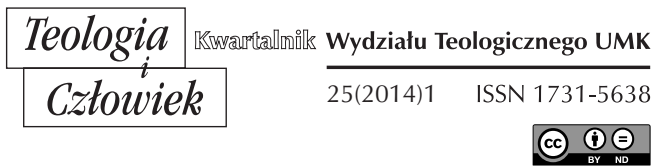

KS. RYSZARD SELEJDAK*

RZYM

\title{
DIACONATO FEMMINILE NELLA CHIESA ANTICA
}

DOI: http://dx.doi.org/10.12775/TiCz.2014.001

\section{INTRODUZIONE}

Dopo il dossier di J. Mayer ${ }^{1}$, gli studi di A. Kahlsbach², R. Gryson³, A.G. Martimort ${ }^{4}$, E. Theodorou ${ }^{5}$, Ch. Bottingheimer ${ }^{6}$, U.E. Eisen ${ }^{7}$, nessuno mette in dubbio che nella Chiesa sino al secolo XII siano esistite donne

* Ks. dr hab. Ryszard Selejdak - prezbiter archidiecezji częstochowskiej, patrysta i teolog dogmatyk. Pracownik watykańskiej Kongregacji Edukacji Katolickiej.

${ }^{1}$ Cfr. J. Mayer, Monumenta de viduis diaconissis virginibusque tractantia, Bonn 1938.

${ }^{2}$ Cfr. A. Kahlsbach, Die altchristliche Einrichtum der Diakonissen bis zu ihren Erlöschen, Freiburg i. Br 1926; Id., Diakonisse, in: Reallexikon für Antike und Christenum, vol. III, Stuttgart 1957, p. 917-928.

${ }^{3}$ Cfr. R. Gryson, Il ministero della donna nella chiesa antica, Roma 1974.

${ }^{4}$ Cfr. A. G. Martimort, Les diaconesses. Essai historique, Roma 1982.

${ }^{5}$ Cfr. E. Theodorou, L'institution des diaconesses dans l'Église orthodoxe et la possibilité de sa rénovation, Contacts 1 (1989) 124-144; Id., Donna e ministero. Presentazione teologica della tradizione e della prassi ecclesiastica bizantina, in: C. Militello (a cura), Donna e ministero, Roma 1991, p. 99-118.

${ }^{6}$ Cfr. Ch. Bottingheimer, Der Diakonat der Frau, "Münchener Theologische Zeitschrift" 47 (1996), p. 253-266.

${ }^{7}$ Cfr. U.E. Eisen, Amtsträgerinnen in frühen Christentum, Göttingen 1996. 
diacono. Le discussioni, come osserva P. Sorci ${ }^{8}$, cominciano quando si tratta di stabilire: l'origine, se cioè questa possa essere attribuita all'epoca apostolica, o si debba collocare nel secolo III con la Didascalia degli Apostoli; l'estensione, cioè se siano state presenti in tutte le Chiese o soltanto in alcune aree geografiche; il valore dell'ordinazione, ossia se si trattava di una semplice benedizione, oppure essa aveva un valore analogo a quella conferita agli uomini, che la teologia successiva chiamerà sacramentale; le prerogative e le mansioni eserciate, se cioè esse sono equiparabili a quelle esercitate dai diaconi. Riguardo le menzionate problematiche esiste una ricca letteratura9.

In questo articolo mi limiterò a presentare il diaconato femminile nella Chiesa antica alla luce del Nuovo Testamento, delle opere di scrittori ecclesiastici, della legislazione dei Concili e della legislazione imperiale dei primi sei secoli.

${ }^{8}$ Cfr. P. Sorci, Donna e diaconato: un'indagine storico-liturgica, in: G. Bellia (a cura), Discernere oggi, le vie, i problemi, le emergenze, Reggio Emilia 1998, p. 98-99.

${ }^{9}$ Cfr. C. Vagaggini, L'ordinazione delle diaconesse nella tradizione greca e bizantina, OCP 40 (1974), p. 145-189; A. L. Szafrański, Diakonise i ich rola w pierwotnym kościele, "Vox Patrum" 9 (1989), p. 737-755; C. Militello, Donna e ministero. Un dibattito ecumenico, Roma 1991; M. Hauke, Überlegungen zum Weihediakonat der Frau, ThGl 77 (1987), p. 108-127; Id., Diakonat der Frau?, Forum Katholische Teologie 12 (1996) 36-45; Id., Der Frauendiakonat als Hebel zur Veränderung der Kirche. Kritische Bilanz einer theologischen Tagung, "Forum Katholische Teologie" 14 (1998), p. 132-147; Id., Il diaconato femminile. Osservazioni sul recente dibattito, "Notitiae" 37 (2001), p. 195-239; M. J. Aubert, Des femmes diacres. Un nouveau chemin pour l'Église, Paris 1987; A. A. Thiermeyer, Der Diakonat der Frau, ThQ1 173 (1993) 226-236; F. Courth, Die Sakramente, Freiburg i. Br. 1995, p. 286-291; A. Miralles, Le diaconesse: bilancio di dodici anni di pubblicazioni (1982-1993), "Ricerche teologiche" 7 (1996), p. 161-172; E, Cattaneo, I ministeri nella Chiesa antica. Testi patristici dei primi tre secoli, Milano 1997, p. 191-194; C. Marucci, Storia e valore del diaconato femminile nella Chiesa antica, "Rassegna di teologia" 38 (1997), p. 771-795; P. Vanzan, Diaconato permanente femminile. Ombre e luci, "La Civiltà Cattolica" 1 (1999), p. 439-452; D. Reininger, Diakonat der Frau in der Einen Kirche. Mit einem Geleitwort von Bischof Karl Lehmann, Stuttgart 1999, p. 76-126; G. L. Müller, Der Empfänger des Weihesakraments. Quellen zur Lehre und Praxis der Kirche, nur Männern das Weihesakrament zu spenden, Würzburg 1999; Id., Priestertum und Diakonat. Der Empfänger des Weihesakramentes in schöpfungstheologischer und christologischer Perspektive, Einsiedeln-Freiburg i. Br. 2000; S. Düren, Diakonat der Frau? Fragen zur Stellung der Frau in der Kirche. Mit einem Geleitwort von Joachim Kardinal Meisner, Buttenwiesen 2000, p. 25-36; P. Zagano, Holy Saturday. An argument for the restoration of the female diaconate in the Catholic Church, New York 2000, p. 87-110; R. Giesen, Können Frauen zum Diakonat zugelassen warden?, Siegburg 2001, p. 26-69. 


\section{IL DIACONATO FEMMINILE IN EPOCA APOSTOLICA E NEL Il SECOLO}

In epoca apostolica, diverse forme di assistenza diaconale agli apostoli e alle comunità esercitate da donne sembrano avere un carattere istituzionale. Così Paolo raccomanda alla comunità di Roma "Febe, nostra sorella, diaconessa ("diakonos") della Chiesa di Cencre" (cfr. Rm 16, 1-4). Benché qui sia usata la forma maschile di "diakonos", non si può concludere che essa indichi già la funzione specifica di "diacono". Da una parte, perché, in questo contesto, "diakonos" significa ancora, in un senso molto generale, servo e, d'altra parte, perché la parola "servo" non ha un suffisso femminile, ma è preceduta da un articolo femminile. Ciò che pare certo è che Febe ha esercitato un servizio nella comunità di Cencre, riconosciuto e subordinato al ministero dell'Apostolo. Si deve osservare che altrove, nei testi di Paolo, le stesse autorità civili sono chiamate "diakonos" (cfr. Rm 13,4) e, come anche lo stesso diavolo è chiamato "diakonos" (cfr. 2 Cor 11, 14-15) ${ }^{10}$.

Gli esegeti sono divisi riguardo a $1 \mathrm{Tm} \mathrm{3,11.} \mathrm{La} \mathrm{menzione} \mathrm{delle}$ "donne" dopo i diaconi può far pensare a donne-diaconi ${ }^{11}$, o alle spose dei diaconi dei quali si è parlato prima. Esse devono essere dignitose, non pettegole, sobrie, fedeli in tutto. Un pò prima Paolo ricorda che le donne non devono insegnare né dirigere gli uomini (cfr. 1 Tm 2, 8-15). Infatti le funzioni di direzione e di insegnamento sono in ogni caso ri-

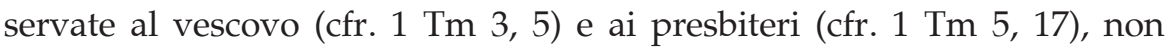
ai diaconi.

Secondo R. Gryson è possibile che una traccia di quelle donne di cui si parla in $1 \mathrm{Tm} \mathrm{3,11}$ si trovi nella celebre Lettera di Plinio il Giovane, governatore della Bitinia, inviata a Traiano riguardante i cristiani ${ }^{12}$. Plinio dichiara che ha giudicato necessario, per conoscere la verità sulla setta dei cristiani, sottoporre alla tortura due serve che venivano chiamate "ministrae": "Necessarium credidi ex duabus ancillis, quae ministrae

${ }^{10}$ Cfr. Commissione Teologica Internazionale, Il Diaconato: evoluzione e prospettive, Città del Vaticano 2003, p. 32-33.

${ }^{11}$ Cfr. P. Sorci, art. cit., p. 99-100; S. Cipriani, Lettere pastorali, in: Nuovissima versione della Bibbia, Torino 19853, p. 513-514, 541-542; L. Swidler, Women Priests. A Catholic Commentary on the Vatican Declaration, Toronto 1977, p. 343. In senso contrario, per esempio, L. Corlay, Diaconesses, qui étiez-vous?, "Le diaconat" 57 (1995), p. 20; E. Cattaneo, I ministeri nella Chiesa antica. Testi patristici dei primi tre secoli, p. 194.

${ }^{12}$ Cfr. R. Gryson, op. cit., p. 42-43. 
dicebantur, quid esset veri et per tormenta quaerere"13. In questa frase "ancilla" è termine che designa la condizione sociale di quelle donne, e "ministra" un titolo che i cristiani attribuiscono loro. E' probabile, anche se non certissimo, che la parola greca corrispondente a "ministra" sia "diakonos"14. Con tutto ciò, non si sa ancora niente di preciso circa lo stato giuridico e le funzioni di quelle donne in seno alla comunità cristiana.

Alla fine del II secolo il testo apocrifo degli Atti di Paolo, presenta Tecla come vera diaconessa, anche se il nome non compare. Essa annunzia la parola di Dio ad Iconio per incarico dell'Apostolo e celebra il sacramento del battesimo o illuminazione ${ }^{15}$.

R. Gryson osserva che gli inizi del diaconato femminile vagano tra tenebre dense, e sembra proprio difficile diradarle ${ }^{16}$.

\section{LE DIACONESSE NELLE CHIESE ORIENTALI TRA IL III E IL VI SECOLO}

Si deve rilevare che i termini specificamente cristiani di "diaconessa" o "diacona" compaiono per la prima volta solamente nel III secolo ${ }^{17}$. Infatti, a partire dal quel secolo, in alcune regioni della Chiesa - e non in tutte - è attestato un ministero ecclesiale specifico, attribuito alle donne chiamate diaconesse ${ }^{18}$. Si tratta della Siria e di Costantinopoli.

\subsection{DIDASCALIA APOSTOLORUM}

Il primo documento ecclesiastico che ricorda le diaconesse nelle regioni della Siria orientale è la Didascalia Apostolorum ${ }^{19}$.

${ }_{13}$ Plinio il Giovane, Lettere, 10, 96, 8, in: P. Carrara (a cura), I pagani di fronte al cristianesimo, Firenze 1984, p. 56.

${ }^{14}$ Cfr. Plinio era governatore della Bitinia, una provincia dell'Asia Minore dove la lingua d'uso era il greco; si può dunque supporre che egli traduca in latino un termine greco.

${ }^{15}$ Cfr. Atti di Paolo, 34.39.43, in: M. Erbetta (a cura), Gli Apocrifi del Nuovo Testamento. Atti e leggende, Casale 1966, p. 256-267.

${ }^{16}$ Cfr. R. Gryson, op. cit., p. 43.

${ }^{17}$ Cfr. Il Diaconato: evoluzione e prospettive, doc. cit., p. 33.

${ }^{18}$ Cfr. A. G. Martimort, op. cit., p. 31.

${ }^{19}$ Cfr. Il Diaconato: evoluzione e prospettive, doc. cit., p. 34. 
La Didascalia si presenta come un'opera pseudepigrafa in quanto usa la finzione letteraria di far parlare direttamente i dodici Apostoli, riuniti a Gerusalemme nell'occasione riportata da At 15. Essi avrebbero prolungato quell'assemblea in cui era presente anche "Giacomo, vescovo di Gerusalemme, fratello del Signore nostro secondo la carne, assieme ai suoi presbiteri e diaconi e a tutta la Chiesa" 20 e avrebbero promulgato questo documento, al fine di contrastare gli pseudo-apostoli e le loro false dottrine ${ }^{21}$. Evidentemente qui l'autore del III secolo proietta sulle origini la struttura ministeriale del suo tempo, cioè la triplice gerarchia "vescovo-presbiteri-diaconi". Questo quadro pseudepigrafo è solo l'opera del redattore finale, il quale molto probabilmente si è servito di materiale più arcaico. Si vede infatti che nel corso del testo la triplice struttura ministeriale non appare mai con quel tipico ordine che si trova già nelle Lettere di Ignazio di Antiochia. Ciò che colpisce maggiormente nella Didascalia è la scarsa attenzione rivolta al ruolo dei "presbiteri", mentre balza in primo piano il binomio "vescovo-diaconi". Inoltre, la Didascalia non solo menziona, ma parla espressamente della necessità delle "donne-diacono", oltre alla ormai tradizionale presenza del gruppo delle "vedove" istituite. Degli altri ordini minori non è detto quasi nulla.

Nella Didascalia la figura del vescovo occupa un posto centrale. Egli ha i tratti di un patriarca biblico onnipotente ${ }^{22}$. $E^{\prime}$ un capo di una piccola comunità, che egli dirige soprattutto con l'aiuto di diaconi e diaconesse. Secondo una tipologia prestata da Ignazio di Antiochia, il vescovo occupa il posto di Dio Padre, il diacono quello di Cristo, la diaconessa quella dello Spirito Santo, mentre i presbiteri rappresentano gli Apostoli e le vedove l'altare ${ }^{23}$. Questa singolare idea che la diaconessa è figura dello Spirito Santo non si incontra in nessun altro luogo, né viene altrove sviluppata. Il fatto che il termine che designa lo Spirito è femminile in siriaco, come anche in ebraico, probabilmente ha facilitato nella coscienza dell'autore siriaco, questa assimilazione a prima vista sorprendente.

${ }^{20}$ Didascalia Apostolorum VI, 12, 3, F. X. Funk, Didascalia et Constitutiones Apostolorum, vol. I, Paderbornae 1905, 327. L'edizione del Funk verrà di seguito abbreviata in DCA. Le traduzioni del testo saranno proprie.

${ }^{21}$ Cfr. ibid., VI, 8, 1, DCA I, 317-318.

${ }^{22}$ Cfr. ibid., II, 33-35, DCA I, 115-121.

${ }^{23}$ Cfr. ibid., II, 26, 4-7, DCA I, 105. 
La Didascalia Apostolorum non fa menzione dell'ordinazione delle diaconesse, mentre descrive le loro funzioni: "Perciò, o vescovo, scegliti degli operai di giustizia, aiutanti che cooperino con te alla vita. Quelli che ti piacciono tra tutto il popolo, li sceglierai e li costituirai diaconi: se è un uomo, perché si occupi delle molte cose necessarie; se è una donna, per il servizio delle donne. Ci sono infatti delle case nelle quali non puoi mandare a delle donne un uomo diacono, a motivo dei pagani, mentre puoi mandare una donna diacono. Ma in molte altre cose è necessario il ministero di una donna diacono. Anzitutto, quando delle donne scendono nell'acqua battesimale, siano unte con l'olio dell'unzione dalla donna diacono. Se poi non si trova presente una donna, e specialmente una che sia diacono, bisogna che sia il battezzante a ungere la battezzata. Ma se $c^{\prime}$ è una donna, e specialmente una che sia diacono, non è conveniente che le donne siano viste dagli uomini, ma il battezzante imponga le mani e unga solo il capo della donna, così come anticamente erano unti i sacerdoti e i re in Israele. Allo stesso modo, anche tu vescovo, imponendo le mani, ungi il capo di coloro che devono essere battezzati, uomini o donne. Quindi, tanto se battezzi tu stesso, quanto se ordini ai diaconi o ai presbiteri di battezzare, sarà la donna diacono, come abbiamo detto prima, a fare l'unzione sulle donne. Sia però l'uomo a pronunciare su di esse l'invocazione dei Nomi divini nell'acqua. Quando colei che è battezzata esce dall'acqua, sia accolta dalla donna diacono, la quale la istruirà e la educherà a custodire l'infrangibile sigillo battesimale nella castità e nella santità. Per questo diciamo che è sommamente richiesto e necessario il ministero di una donna diacono. Infatti anche il nostro Signore e Salvatore era servito da donne: Maria Maddalena, Maria figlia di Giacomo e madre di Giuseppe, e la madre dei figli di Zebedeo, assieme ad altre donne. Anche per te, o vescovo, è necessario il ministero delle donne diacono in molte cose. Infatti, nelle case dei pagani dove ci sono donne credenti, è necessaria una donna diacono per entrare e visitare quelle che sono malate, perché serva loro ciò che occorre e lavi quelle che hanno iniziato a riprendersi dalla malattia. I diaconi imitino i vescovi nelle loro azioni; ma siano ancora più esercitati nella pratica, e non bramino un guadagno disonesto, per svolgere bene il loro ministero. Saranno in numero sufficiente, secondo la moltitudine di cui è composta la Chiesa, così che possano soddisfare le esigenze delle donne più anziane, ormai debilitate, e di quei fratelli e sorelle che sono nella malattia, adempiendo con prontezza il loro ministero. La donna diacono sia sollecita verso le donne, mentre il 
diacono che è uomo, verso gli uomini; e sia prontissimo a recarsi dove il vescovo comanda per il ministero e il servizio" 24 .

La posizione della diaconessa è presentata in questo testo come analoga a quella del diacono: gli uomini e le donne diaconi sono collaboratori del vescovo, scelti liberamente da lui tra il popolo ed istituiti tali per assisterlo nel suo compito pastorale. Questa analogia è indicata chiaramente dal termine che designa la diaconessa: "donna diacono" o semplicemente "diacono" usato come nome femminile. Non è evidente che le attribuzioni della diaconessa siano numerose come quelle del diacono maschile. Il diacono, si legge, deve occuparsi di molte cose che sono necessarie ${ }^{25}$, deve essere pronto ad eseguire tutti gli ordini che gli impartisce il vescovo ${ }^{26}$. Egli amministra i beni della comunità in nome del vescovo e, come il vescovo, è mantenuto da essa. In altri testi appare ancora più chiaramente che il diacono è braccio destro del vescovo, suo orecchio, sua bocca, intermediario tra lui e i fedeli ${ }^{27}$. Assiste il vescovo durante la celebrazione dell'Eucaristia e cura l'ordine nell'assemblea ${ }^{28}$. Niente di tutto questo è detto a proposito della diaconessa. Il menzionato servizio alle donne si riduce solamente in due ambiti. Da una parte il servizio per le donne credenti e le ammalate, presentato come suppletivo di quello del diacono maschile nel caso che costui non possa essere mandato in casa di cristiani senza che i pagani se ne abbiano a male ${ }^{29}$. D'altra parte è una diaconessa che, per fare bene le cose, dovrebbe ungere sul corpo le donne prima che scendano nell'acqua battesimale, ed accoglierle quando ne escano, perché è sconveniente che la nudità delle donne sia vista dagli uomini ${ }^{30}$. L'autore della Didascalia insiste sull'utilità, anzi sulla necessità del ministero in questione. Ricorda che nostro Signore stesso ha goduto del servizio di donne durante la sua esistenza terrena. Ma è ovvio che le diaconesse non stanno dappertutto. In mancanza di diaconesse, una donna qualsiasi, e in mancanza di donne, lo stesso ministro di sesso maschile del battesimo farà alle donne l'unzione sul corpo. In

${ }^{24}$ Ibid., III, 12, 1 - 13, 1, DCA I, 208-214.

${ }^{25}$ Cfr. ibid., III, 12, 1, DCA I, 208.

${ }^{26}$ Cfr. ibid., III, 13, 1, DCA I, 214.

27 Cfr. ibid., II, 28, 6, DCA I, 108-110.

${ }^{28}$ Cfr. ibid., II, 57, 6-7, DCA I, 161-163.

${ }^{29}$ Cfr. G. Schöllgen, Die Anfänge der Professionalisierung des Klerus und das Amt in der syrischen Didaskalie, Münster 1997, p. 167-172; P. Hofrichter, Diakonat und Frauen im kirchlichen Amt, Heiliger Dienst 50 (1996) 149.

${ }^{30}$ Cfr. P. Hofrichter, art. cit., p. 149. 
ogni caso, questo è tutto quello che è concesso alle diaconesse. Mentre il diacono può essere delegato dal vescovo per amministrare il battesimo, non c'è dubbio che la diaconessa ricade sotto la proibizione che vieta alle donne di battezzare; tanto è vero che viene fatta la precisazione che anche quando la diaconessa è presente e fa alle donne l'unzione sul corpo, è sempre un uomo che deve pronunciare la formula battesimale. Ancora, la diaconessa è senza dubbio compresa nel divieto fatto alle donne di insegnare, eccetto l'istruzione che essa è invitata a rivolgere alle neofite uscenti dall'acqua battesimale. In poche parole, si ha l'impressione di trovarsi di fronte ad un ministero stabilito soltanto in alcuni luoghi, le cui attribuzioni restano però strettamente limitate ${ }^{31}$.

\subsection{IL CANONE 19 DEL PRIMO CONCILIO DI NICEA}

Il canone 19 del Primo Concilio di Nicea, del 325, prescrive quanto segue: "Quanto ai paulianisti, che intendono passare alla chiesa cattolica, bisogna osservare l'antica prescrizione che essi siano senz'altro ribattezzati. Se qualcuno di essi, in passato, era appartenuto al clero, purché del tutto irreprensibile, una volta ribattezzato potrà essere ordinato dal vescovo della chiesa cattolica. Ma se l'esame dovesse far concludere che si tratta di indegni, è bene deporli. Questo modo d'agire sarà usato anche con le diaconesse e, in genere, con quanti hanno un ministero nella chiesa. Quanto alle diaconesse che sono nella stessa situazione, in particolare ricordiamo che esse, non avendo ricevuto alcuna imposizione delle mani, devono essere computate senz'altro tra i laici" ${ }^{\prime 2}$.

Il suddetto testo tratta della reintegrazione dei paulianisti, cioè dei discepoli di Paolo di Samosata, i quali professavano una dottrina erronea in materia trinitaria. Per questa ragione, i Padri niceni giudicano invalido il loro battesimo e stabiliscono di ribattezzarli qualora ritornino alla Chiesa cattolica. Quanto ai chierici ordinati in quella setta, la loro ordinazione non può risultare che invalida, allo stesso titolo. Il Concilio tuttavia accetta di mantenerli nelle loro funzioni, per mezzo di una riordinazione fatta dal vescovo cattolico, sotto condizione che non ci sia di che lamentarsi di loro per altri motivi; diversamente, saranno deposti. La stessa regola, aggiunge

${ }^{31}$ Cfr. R. Gryson, op. cit., p. 88-89; P. Sorci, art. cit., pp. 100-101.

${ }^{32}$ Primo Concilio di Nicea, can. 19, in: G. Alberigo-G. L. Rossetti-P. P. JoannouC. Leopardi-P. Prodi (a cura), Conciliorum Oecumenicorum Decreta, Bologna 1996, p. 15. 
il testo, sarà applicata alle diaconesse e, in generale, a tutti quelli che figurano nel canone, cioè nell'elenco delle persone che compiono una funzione all'interno delle strutture determinate dalla legislazione ecclesiastica e che, a questo titolo, ricevono un sussidio dalla Chiesa. Come osserva R. Gryson, questa frase è stata spesso interpretata in modo erroneo. La regola in questione dice che se la persona in esame è degna di esercitare la funzione che esercitava nell'eresia, essa potrà esercitarla in futuro. Questa regola viene confermata dall'espressione "così pure" all'inizio della frase. Il testo non parla di riordinazione a proposito delle diaconesse e di quelli che figurano nel canone. Il fatto di menzionare costoro a parte, in un secondo tempo, indica proprio che il loro è un caso un poco diverso. Molti autori, per non aver notato ciò e per aver voluto estendere l'analogia della regola fino alla necessità di una riordinazione anche per le diaconesse, sono incorsi in una difficoltà insormontabile. Il testo infatti afferma, subito dopo, che le diaconesse non ricevono nessuna imposizione di mani e appartengono comunque al numero dei laici. Per evitare questa contraddizione, quegli autori hanno architettato ipotesi di ogni genere, più complicate, più indimostrabili. Non vale la pena qui riportarle e discuterle. Si possono trovare, riassunte con chiarezza e puntualmente criticate, in uno studio approfondito di A. Kalsbach sul canone 19 di Nicea ${ }^{33}$. In realtà la contraddizione esiste solo nella mente di quegli autori. Il Concilio infatti non voleva affermare in nessun modo che le diaconesse devono essere riordinate, come gli altri chierici, qualora fossero mantenute nelle loro funzioni a motivo di una condotta irreprensibile: ciò sarebbe chiaramente in contraddizione con l'affermazione che esse non ricevono nessuna ordinazione. Infatti si afferma che esse, come i chierici, potranno essere mantenute nelle loro funzioni qualora la loro condotta risultasse irreprensibile. Questo vale nonostante esse non ricevano nessuna ordinazione e il loro caso, di conseguenza, sarà diverso da quello dei chierici. Esse infatti appartengono al numero dei laici.

\subsection{CANONI DI BASILIO}

Le tre lettere cosiddette "canoniche" che S. Basilio mandò ad Anfilochio di Iconio, contengono un certo numero di regole concernenti le diaconesse.

${ }^{33}$ Cfr. A. Kalsbach, Die Diakonissenweihe in Kan. 19 der Konzils von Nicäe, Römische Quartalschrift 32 (1924), p. 166-169. 
Il canone 44 dichiara innanzitutto che "la diaconessa che ha fornicato con un pagano può essere ammessa alla penitenza; costei sarà ammessa all'offerta nel settimo anno, a condizione, ben inteso, che abbia vissuto nel frattempo in castità" ${ }^{34}$. Non si può dire se questa pena sia più o meno pesante di quella che veniva imposta ai fornicatori in generale. Secondo il canone 22, la penitenza di costoro è di quattro anni ${ }^{35}$, mentre secondo il canone 59, è di sette anni, e i colpevoli sono riammessi alla comunione solo nell'ottavo anno ${ }^{36}$. In entrambi i casi, viene precisato che devono passare attraverso tutti i gradi della penitenza pubblica. Può darsi che la diaconessa sia esentata da quest'ultima esigenza, almeno se si intende il termine "metanoia" ("mutamento", "correzione", "pentimento", "penitenza") del canone 44 in senso tecnico. In effetti, questo termine designa a volte la terza delle quattro classi di penitenti, come si può vedere nel canone 22. L'insieme del processo penitenziale è designato qui con il termine "epitimesis" ("censura", "biasimo", "accusa", "rimprovero") ${ }^{37}$. Come che sia, per il fatto che la diaconessa colpevole sia privata della comunione e costretta a penitenza, esso indica chiaramente che non è assimilata ai membri del clero. Difatti, i chierici di qualsiasi grado che si sono macchiati di una colpa grave $\mathrm{e}^{38}$, e in particolare il diacono fornicatore $^{39}$, vengono deposti ma non scomunicati, in virtù del principio che non bisogna punire due volte la stessa colpa.

Non si vede bene il legame logico che unisce la prima frase del canone 44, con la seconda, a parte il fatto che nei due casi si parla di "pagano". "Il pagano che, dopo aver avuto la fede, passa nuovamente all'empietà, ritorna al suo vomito" ${ }^{\prime 4}$. Poi si parla di nuovo della diaconessa: "Quanto a noi, aggiunge Basilio, non permettiamo che il corpo della diaconessa, visto che è consacrato, serva ancora ad un uso carnale"41.

${ }^{34}$ Basilio di Cesarea, Lettere ad Anfilochio sui canoni, p. 44, P. P. Joannou, Discipline générale antique (IVe-IXe s.). Les Canons des Synodes Particuliers, vol. 1-2, Grottaferrata 1962, 2, 136, 3-7. L'edizione del Joannou verrà di seguito abbreviata in Joannou. Le traduzioni del testo saranno proprie.

${ }^{35}$ Cfr. ibid., 22, Joannou, 125, 3-13.

${ }^{36}$ Cfr. ibid., 59, Joannou, 146, 7-12.

${ }^{37}$ Cfr. ibid., 22, Joannou, 125, 5 e 9-10.

${ }^{38}$ Cfr. ibid., 32, Joannou, 131, 10-14; 142, 9-15.

${ }^{39}$ Cfr. ibid., 3, Joannou, 100, 11-101, 3.

40 Ibid., 44, Joannou, 136, 7-9.

${ }^{41}$ Ibid., Joannou, 136, 9-12. 
Sembra che Basilio, come fa più di una volta nei suoi canoni ${ }^{42}$, esprima qui il suo pensiero personale o la regola alla quale lui stesso si attiene, dopo aver enunciato inizialmente la regola tradizionale. Se questa interpretazione è esatta, Basilio imporrebbe alla diaconessa una regola molto più esigente di quella espressa nella prima frase del canone 44 . Quest'ultima colpisce soltanto l'unione illecita di una diaconessa con un pagano; non contempla il caso di un matrimonio regolare con un cristiano. Per Basilio la diaconessa è tenuta alla continenza. Il suo corpo, poiché è consacrato, non può più servire ad un uso carnale. Ci si chiede: cos'è che rende il corpo della diaconessa "consacrato"? Forse l'ordinazione? Il termine qui usato "kathieromenon" ("dedicato", "offerto") non significa in nessun modo l'ordinazione, contrariamente a quanto vuole insinuare A. Kalsbach ${ }^{43}$. E' possibile che il corpo della diaconessa venga detto "consacrato" a causa del voto di continenza che si è richiesto da lei. In un altro canone, Basilio parla della vergine come di un "vaso sacro dedicato al Signore" ${ }^{\prime 4}$. Il canone 44 è l'unico in cui Basilio faccia menzione della diaconessa. Non si può dunque dire altro circa la condizione giuridica e le funzioni delle diaconesse nell'ambiente della Cappadocia di quel periodo.

\subsection{IL CANONE 11 DEL CONCILIO DI LAODICEA}

Le collezioni canoniche greche attribuiscono ad un Concilio che si sarebbe tenuto negli anni 363-364 a Laodicea di Frigia Pacatiana, una serie di 59 canoni, inseriti, da una parte tra i canoni di Antiochia o di Serdica, dall'altra tra i canoni di Costantinopoli. La forma stereotipata, e spesso molto breve, con la quale questi canoni del Concilio di Laodicea si presentano, oltre alla somiglianza contenutistica di molti di essi con i canoni di concili anteriori, danno l'impressione che si tratti piuttosto di titoli o di condensati che non del testo integrale di disposizioni canoniche originali.

La formulazione lapidaria dei suddetti canoni evidentemente non ne facilita l'interpretazione. Questo succede in modo particolare con il

${ }^{42}$ Per esempio, cfr. ibid., 3. 4. 18. 19. 47, ecc.

${ }^{43}$ Cfr. A. Kalsbach, Die altkirchliche Einrichtung der Diakonissen bis zu ihrem Erlöschen, Friburgo i.Br 1926, p. 109, nota 1.

${ }^{44}$ Basilio di Cesarea, Lettere ad Anfilochio sui canoni, 18, Joannou, 2, 119, 27-28. 
canone 11 che prescrive: "Non si devono costituire nella Chiesa quelle che si chiamano "anziane» o "presidenti»" 45 .

Secondo alcuni, il termine "presbytis" ("presidente") designerebbe in questo canone le diaconesse ${ }^{46}$, e il Concilio protesterebbe contro il fatto che esse ricevono un'ordinazione ${ }^{47}$. Ma in nessun documento in cui si parli di diaconesse si può trovare che esse occupino un grado o esercitano delle funzioni che possono giustificare questo titolo di "anziane" o di "presidenti". Inoltre il termine usato abitualmente, a partire dalla fine del secolo IV, per designare il rito di entrata in carica delle diaconesse, è "cheirotonein", che significa precisamente "ordinare", non "kathistasthai" che significa più genericamente "installare", "costituire". Altri pensano che si tratti di diaconesse più vecchie delle altre o di diaconesse di grado superiore $^{48}$. Ciò risolverebbe, fino a un certo punto, la prima delle obiezioni esposte, non la seconda; e del resto, si cercherebbe inutilmente in altri testi qualche traccia di gerarchia all'interno dell'ordine delle diaconesse. P. H. Lafontaine ritiene che non si tratti di diaconesse, ma di vedove, più precisamente di vedove "istituite", come si è visto nella Didascalia Apostolorum. Esse, in un certo senso, rappresentano una classe superiore all'interno dell'insieme delle vedove. Il Concilio di Laodicea intenderebbe abolire questa istituzione ${ }^{49}$. Secondo R. Gryson questa interpretazione è più plausibile ${ }^{50}$. Più che alla Didascalia Apostolorum, essa potrebbe rifarsi al Testamento di nostro Signore Gesu Cristo, nel quale più di una volta si parla di vedove che hanno la precedenza, che sono ugualmente chiamate "anziane". Purtroppo il testo originale del Testamento è perduto ed esistono solo versioni in varie lingue orientali, di modo che non si può dire se alla parola che si traduce con "anziane" corrispondeva in greco "presbyterai" ("presidenti") ${ }^{51}$.

${ }^{45}$ Concilio di Laodicea, can. 11, Joannou, 1-2, 135, 618.

${ }^{46}$ Cfr. J. Forget, Diaconesses, in: Dictionnaire de théologie catholique, vol. 4, Paris 1903-1972, p. 688; H. Leclercq, Diaconesse, in: Dictionnaire d' archéologie chrétienne et de liturgie, vol. 4, Paris 1924-1953, p. 725.

${ }^{47}$ Cfr. P. De Labriolle, «Mulieres in Ecclesia taceant». Un aspect de la lutte antimontaniste, Bulletin d'ancienne littérature et d'archéologie chrétiennes 1 (1911) , p.116-117.

${ }^{48}$ Cfr. K. J. Hefele, Histoire des conciles, vol. 1-2, Paris 1907, p. 1003.

${ }^{49}$ Cfr. P. H. Lafontaine, Les conditions positives de l'accession aux orders dans la premičre legislation ecclésiastique, Ottawa 1963, p. 39, nota 99.

${ }^{50}$ Cfr. R. Gryson, op. cit., p. 107.

${ }^{51}$ Cfr. ibid. 


\subsection{LE COSTITUZIONI APOSTOLICHE}

Le Costituzioni apostoliche sono la più vasta compilazione canonica e liturgica dell'antichità cristiana. Esse hanno esercitato un influsso durevole sulla disciplina delle ordinazioni in Oriente, benché non siano state mai considerate ufficiali. I sei primi libri consistono in un rimaneggiamento della Didascalia Apostolorum, infarcito di aggiunte. La prima metà del libro 7 è un ampliamento della Didaché. La seconda metà contiene formulari di preghiere e prescrizioni concernenti l'istruzione dei catecumeni e il battesimo. La parte più preziosa dell'opera è il libro 8 , che utilizza come fonte la Tradizione apostolica di Ippolito. Propone, tra l'altro, un rituale per le diverse ordinazioni e, in questa occasione, descrive la liturgia eucaristica. Alla fine del libro 8 , si trovano 85 canoni, conosciuti sotto il nome di Canoni degli apostoli. Le Costituzioni e i Canoni rappresentano la tradizione siriana e risalgono alla fine del IV secolo. L'autore anonimo è certamente lo stesso della lunga recensione interpolata delle Lettere di Ignazio di Antiochia. Il raffronto tra le Costituzioni apostoliche e le relative fonti mostra quanto l'istituzione delle diaconesse si sia evoluta durante la seconda metà del III secolo e tutto il secolo successivo ${ }^{52}$.

R. Gryson ${ }^{53}$ osserva che di diaconesse, se si eccettua una breve menzione nella tipologia della Chiesa ${ }^{54}$, si parla solo in un passo della Didascalia Apostolorum, dove vengono indicate le funzioni per le quali la loro collaborazione risulta utile al vescovo ${ }^{55}$. Questo passo è ripreso e rimaneggiato in più di un punto dalle Costituzioni, che del resto anche in altri luoghi si riferiscono alle diaconesse. Il termine usato per designarle è "diakonos" nei passi paralleli alla Didascalia dei libri 2 e 3. Invece, in un capitolo proprio delle Costituzioni, nel libro 6, e nel libro 8, viene adoperato il termine "diakonissa" 56 .

Secondo la Didascalia, il vescovo sceglie per farsi assistere nel suo ministero pastorale, quelli che vuole tra tutto il popolo, e li costituisce diaconi, gli uomini per l'esecuzione delle numerose cose che sono necessarie, le donne per il servizio riguardante le donne ${ }^{57}$. Secondo le Costitu-

${ }^{52}$ Cfr. ibid., p. 109.

${ }^{53}$ Cfr. ibid.

${ }^{54}$ Cfr. Didascalia Apostolorum II, 26, 6, DCA I, 86.

${ }_{55}$ Cfr. ibid., III, 12, 1-13, 1, DCA I, 86-88.

${ }^{56}$ Cfr. ibid.

${ }^{57}$ Cfr. ibid., III, 12, 1, DCA I, 208. 
zioni, solo i diaconi di sesso maschile possono essere scelti dall'insieme del popolo ${ }^{58}$. La diaconessa, invece, deve essere una vergine pura $\mathrm{o}$, in mancanza di questa, una vedova sposata una volta sola ${ }^{59}$. Le diaconesse, dunque, vengono reclutate tra i due gruppi di donne di cui si è parlato precedentemente, quello delle vergini, preferibilmente, o altrimenti quello delle vedove. L'utilità primaria attribuita al loro ministero è la stessa che nella Didascalia: vi sono case nelle quali il vescovo non può mandare un diacono senza dare occasione di maldicenze ${ }^{60}$. Ciò sta a indicare che anche qui una delle funzioni principali delle diaconesse resta l'assistenza e la cura a domicilio delle donne inferme o incapaci, benché le Costituzioni abbiano soppresso una frase in cui, un po' più avanti, si fa parola più espressamente di questo servizio, come del resto accade spesso quando la Didascalia presenta delle ripetizioni ${ }^{61}$. Ma oltre a ciò le Costituzioni, ben più della Didascalia, sembra vogliano esibire tutto l'assortimento delle funzioni della diaconessa. Infatti, la Didascalia, dopo aver prescritto che i diaconi devono essere in numero sufficiente affinché tutti gli infermi abbiano assistenza, diceva che le donne devono occuparsi preferibilmente del servizio femminile, gli uomini del servizio degli uomini, e che inoltre i diaconi maschili devono essere pronti a compiere tutte le altre missioni che il vescovo giudicasse utile dover loro affidare ${ }^{62}$. Il ministero delle diaconesse comporta dunque qui la sola cura delle donne, mentre quello dei diaconi implica la cura degli uomini e altri eventuali compiti affidati loro dal vescovo. Secondo le Costituzioni, invece, gli uni e le altre (uomini diaconi e donne diaconi) possono essere incaricati di portare messaggi, eventualmente anche fuori città, ed anche di altri servizi ${ }^{63}$. L'autore non dice in che cosa consistono questi altri servizi. Si può solo notare che le Costituzioni ipotizzano una doppia conseguenza del rapporto tipologico proposto dalla Didascalia tra la diaconessa e lo Spirito Santo. La prima è che, come il Paraclito, secondo il discorso di addio fatto da Gesù, non fa niente e non dice niente da se stesso ma "glorifica" Cristo eseguendo la sua volontà, così la diaconessa "tipo" dello Spirito Santo, non deve fare o dire niente senza il diacono, "tipo" di Cristo. La seconda è che, come

\footnotetext{
${ }^{58}$ Cfr. Costituzioni apostoliche III, 16, 1, DCA I, 209.

${ }^{59}$ Cfr. ibid., VI, 17, 4, DCA I, 341.

${ }^{60}$ Cfr. ibid., III, 16, 1, DCA I, 209.

${ }^{61}$ Cfr, Didascalia Apostolorum III, 12, 4, DCA I, 201.

${ }^{62}$ Cfr. ibid., III, 13, 1, DCA I, 87-88.

${ }^{63}$ Cfr. Costituzioni apostoliche III, 19, 1, DCA I, 215.
} 
non è possibile credere in Cristo senza l'ammaestramento dello Spirito, così nessuna donna deve andare a trovare il vescovo o il diacono senza la diaconessa ${ }^{64}$. Sembra dunque che quest'ultima svolga un compito di intermediario tra le donne e i membri maschili della gerarchia, e forse, secondo un'usanza di cui abbiamo traccia altrove ${ }^{65}$, doveva essere testimone della correttezza dei loro colloqui.

Quanto al ruolo liturgico della diaconessa, la Didascalia non parlava d'altro che dell'assistenza al momento dell'amministrazione del battesimo alle donne ${ }^{66}$. Anche qui vengono fuori differenze significative tra le Costituzioni e la loro fonte. Senza citare le modifiche o le precisazioni apportate allo stesso rituale del battesimo, è interessante constatare che le Costituzioni non contemplano più il caso in cui, non essendo presente una diaconessa, tocca a una donna qualsiasi o anche allo stesso ministro del battesimo fare l'unzione sul corpo delle donne ${ }^{67}$. Dunque nell'ambiente delle Costituzioni il ministero delle diaconesse avrebbe conosciuto, nel frattempo, una larga diffusione. E' questo indubbiamente il motivo, per cui l'autore di esse non trova necessario giustificare l'esistenza di tale ministero con l'esempio delle donne che servivano Gesù. D'altro canto, l'autore delle Costituzioni reagisce all'idea che una donna possa, per quanto minimamente, avere un incarico che riguardi l'insegnamento in chiesa, e là dove la Didascalia prescrive che la diaconessa accolga le donne quando escono dalla vasca battesimale e le istruisca dicendo loro che il sigillo del battesimo deve essere conservato intatto nella santità e nella castità, le Costituzioni scrivono semplicemente che all'uscire dal fonte gli uomini devono essere accolti dal diacono e le donne dalle diaconesse, affinché il conferimento del sigillo, che dovrà essere mantenuto intatto, avvenga in maniera degna e decente ${ }^{68}$.

Accanto a questo compito nella celebrazione del battesimo, che era solo occasionale, le Costituzioni assegnano alla diaconessa un compito più regolare: l'accoglienza delle donne nelle assemblee liturgiche. Come il diacono fa con gli uomini, così esse devono badare a che le donne in chiesa trovino un posto, siano esse povere o ricche, ed eventualmente

\footnotetext{
${ }^{64}$ Cfr. Costituzioni apostoliche II, 26, 6, DCA I, 105.

${ }^{65}$ Cfr. per esempio S. Girolamo, Lettere, 52, 5, 6, CSEL 54, 424, 3-7.

${ }^{66}$ Cfr. Didascalia Apostolorum III, 12, 2-3, DCA I, 208-210.

${ }^{67}$ Cfr. ibid., III, 12, 2, DCA I, 208-214; Costituzioni apostoliche III, 16, 2, DCA I,
} 209-211.

${ }^{68}$ Cfr. ibid., III, 12, 3, DCA I, 210; Costituzioni apostoliche III, 16, 4, DCA I, 211. 
a far sì che le più giovani cedano il proprio posto alle più vecchie ${ }^{69}$. Indubbiamente, il riferimento alle donne incaricate di custodire le porte della tenda della testimonianza e del tempio, nella preghiera di ordinazione delle diaconesse ${ }^{70}$, allude a questa funzione. Le Costituzioni insistono sul fatto che il ministero liturgico delle diaconesse si limita a questo: "La diaconessa non benedice e non compie nulla di ciò che fanno i presbiteri e i diaconi, ma vigila le porte e assiste i presbiteri in occasione del battesimo delle donne, per ragioni di decenza"71.

Nonostante la somiglianza dei nomi e nonostante il fatto che la diaconessa sia presentata come il corrispondente femminile del diacono maschio, le sue funzioni non sono dunque identiche in tutto. In particolare la diaconessa non assiste il vescovo e i sacerdoti all'altare e non aiuta a distribuire la comunione.

Secondo le Costituzioni le diaconesse occupano un posto assai importante in seno alla comunità ecclesiale. Esse hanno una posizione di preminenza tra le donne e vengono prima delle vergini e delle vedove che non sono investite di questa funzione. Fanno la comunione prima delle altre vergini e delle vedove ${ }^{72}$ e, nelle enumerazioni, sono citate sempre prima di esse ${ }^{73}$. Per di più: là dove la Didascalia dice che le vedove devono essere soggette ai vescovi e ai diaconi, le Costituzioni aggiungono: "Come pure alle diaconesse" ${ }^{\prime \prime 7}$. Inoltre, le Costituzioni inseriscono le diaconesse nel clero. Ciò scaturisce chiaramente dal fatto che esse, con i chierici e con loro soltanto, partecipano alla distribuzione dei pani che sono risultati in soprappiù tra quelli offerti dai fedeli per l'Eucaristia e che non sono stati consacrati ${ }^{75}$. Secondo le Costituzioni le diaconesse ricevono, come gli altri chierici, un'ordinazione conferita per mezzo dell'imposizione delle mani con epiclesi dello Spirito Santo ${ }^{76}$, mentre vergini e vedove non sono ordinate $^{77}$. Questa ordinazione, come tutte le altre, è un rito strettamente

${ }^{69}$ Cfr. Costituzioni apostoliche II, 58, 4-6, DCA I, 169-171.

${ }^{70}$ Cfr. ibid., VIII, 20, 1, DCA I, 524.

${ }^{71}$ Ibid., VIII, 28, 6, DCA I, 530.

${ }^{72}$ Cfr. ibid., VIII, 13, 14, DCA I, 516.

${ }^{73}$ Cfr. ibid., II, 26, 3, DCA I, 103-105.

${ }^{74}$ Cfr. Didascalia Apostolorum III, 8, 1, DCA I, 196; Costituzioni apostoliche III, 8,

1, DCA I, 197.

${ }^{75}$ Cfr. Costituzioni apostoliche VIII, 31, 2, DCA I, 532-534.

${ }^{76}$ Cfr. ibid., VIII, 19, 2, DCA I, 524.

77 Cfr. ibid., VIII, 24, 2, DCA I, 528. 
riservato al vescovo, e i presbiteri non possono conferirla ${ }^{78}$. Essa avviene alla presenza dei presbiteri, dei diaconi e delle diaconesse ${ }^{79}$. La preghiera pronunciata dal vescovo durante l'ordinazione è la seguente: “Dio, eterno, Padre di Nostro Signore Gesù Cristo, creatore dell'uomo e della donna, tu che hai riempito di spirito Myriam, Debora, Anna e Ulda, che non hai giudicato indegno che tuo Figlio, l'Unigenito, nascesse da una donna, tu che nella tenda della testimonianza e nel tempio hai istituito custodi per le tue porte sante, tu stesso guarda ora la tua serva qui presente, proposta per il diaconato, donale lo Spirito Santo e purificala da ogni impurità della carne e dello spirito, perché compia degnamente l'ufficio che le è stato affidato, per la tua gloria e a lode del tuo Cristo, da cui a te gloria e adorazione nello Spirito Santo, per i secoli. Amen"80.

Questa preghiera ha la medesima forma di quella delle altre ordinazioni ed anche il contenuto è analogo. Come modelli biblici, ricorda le profetesse dell'Antico Testamento, perché donne piene di Spirito Santo, nonché le custodi delle porte del tempio. Ricorda anche che il Figlio unico di Dio è nato da una donna. Poi chiede per l'eletta il dono dello Spirito Santo, sia come grazia di santità personale che come aiuto per adempire il ministero.

In un testo del libro 3, nell'elenco delle ordinazioni riservate al vescovo, le diaconesse vengono subito dopo i diaconi, prima degli altri chierici ${ }^{81}$. Essendo probabilmente comprese nella formula della preghiera dei fedeli: "Per tutto il diaconato e il ministero in Cristo", vengono di conseguenza, anche qui, prima dei lettori e dei cantori ${ }^{82}$. In altri elenchi, invece, la diaconessa non figura immediatamente dopo il diacono, ma dopo l'insieme dei componenti maschili del clero $^{83}$. In questi casi essa fa la comunione solo dopo il clero e gli "asceti", cioè i monaci e gli uomini votati alla continenza ${ }^{84}$. La posizione della diaconessa all'interno della gerarchia dei chierici appare dunque incerta e poco determinata: la somiglianza del nome e quella almeno parziale delle funzioni invita-

\footnotetext{
${ }^{78}$ Cfr. ibid., III, 11, 3, DCA I, 201.

${ }^{79}$ Cfr. ibid., VIII, 16, 2, DCA I, 522.

${ }^{80}$ Ibid., VIII, 20, 1-2, DCA I, 524.

${ }^{81}$ Cfr. ibid., III, 11, 3, DCA I, 201.

${ }^{82}$ Cfr. ibid., VIII, 10, 9, DCA I, 490.

${ }^{83}$ Cfr. ibid., II, 26, 3, DCA I, 103-104; VIII, 28, 2-6, DCA I, 530; VIII, 28, 7, DCA I, 530; VIII, 28, 8, DCA I, 530; VIII, 31, 2, DCA I, 532-534.

${ }^{84}$ Cfr. ibid., VIII, 13, 14, DCA I, 516.
} 
va naturalmente ad accostarla al diacono. In questo senso, si esitava ad accordare a delle donne la preminenza sugli uomini ${ }^{85}$.

\subsection{IL CANONE 15 DEL CONCILIO DI CALCEDONIA}

Il Concilio di Calcedonia (451) ha trattato delle diaconesse nel suo 15 canone. Esso prescrive: "Una donna non deve essere ordinata diaconessa prima dei quaranta anni, e non lo dovrà essere che dopo una severa inchiesta. Se dopo aver ricevuto l'ordinazione ed aver esercitato il ministero per qualche tempo, arrivi a sposarsi, oltraggiando la grazia di Dio, che sia solennemente scomunicata insieme a colui che si è unito ad essa" ${ }^{\prime \prime 6}$.

La prima cosa da notare è che la diaconessa, come già lo era nelle Costituzioni apostoliche, riceve l'ordinazione con l'imposizione delle mani: ciò risulta dall'uso del verbo "cheirotonein" ("ordinare") e del sostantivo "cheirotonia" ("ordinazione"), che negli atti e nei canoni del Concilio di Calcedonia hanno di norma questo senso. Il limite di età di quaranta anni sembra che sia il risultato di un compromesso. La legislazione anteriore tendeva, a quanto sembra, ad essere più severa e a rifiutare l'accesso agli ordini a donne ancora relativamente giovani. Essa esigeva sessanta anni, età simbolica della vecchiaia nell'antichità, come accadeva per l'iscrizione nel numero delle vedove, secondo la $1 \mathrm{Tm}$. In realtà erano state ammesse nei ranghi delle diaconesse donne notoriamente più giovani, la più celebre delle quali è certamente Olimpia. Il limite di quaranta anni si rivela così come un termine medio ${ }^{87}$. Alla ricezione dell'ordine è connesso, per la diaconessa e per gli altri chierici, il divieto di contrarre matrimonio. Se violerà questa proibizione, sarà anatematizzata per aver "oltraggiato la grazia di Dio". Il Concilio di Calcedonia si mostra più severo verso le diaconesse che verso le vergini consacrate che mancano ai voti. Invece di scagliare contro costoro l'anatema dice solo che saranno private della comunione, e soprattutto autorizza il vescovo ad essere indulgente nei loro riguardi, cosa che non si verifica per le diaconesse ${ }^{88}$.

${ }^{85}$ Cfr. R. Gryson, op. cit., p. 125.

${ }^{86}$ Concilio di Calcedonia, can. 15, Joannou, 1-1, 81, 12 - 82, 4.

${ }^{87}$ Cfr. R. Gryson, op. cit., p. 127.

${ }^{88}$ Cfr. Concilio di Calcedonia, can. 16, Joannou, 1-1, 82, 8-15. 


\subsection{IL TESTAMENTO DI NOSTRO SIGNORE GESÙ CRISTO}

Il Testamento di Nostro Signore Gesù Cristo è un'opera apparsa nella seconda metà del secolo $\mathrm{V}$, probabilmente in un ambiente monofisita della Siria. E' stato scritto in greco, ma sono state conservate solo le versioni in siriaco, arabo ed etiopico. Si ispira alla Tradizione apostolica di Ippolito, che rimaneggia ed ampia considerevolmente ${ }^{89}$.

Secondo il Testamento di Nostro Signore Gesủ Cristo le diaconesse costituiscono un grado nettamente inferiore rispetto a quello delle vedove. In chiesa il loro posto è vicino alla porta principale dell'edificio ${ }^{90}$. Durante l'offerta del sacrificio eucaristico stanno all'interno della Tenda, ma all'ultimo posto, dopo i lettori e i suddiaconi ${ }^{91}$. Nella litania diaconale si fa menzione di esse, assieme ai suddiaconi e ai lettori, però dopo di loro ${ }^{92}$. Dopo queste due ultime indicazioni potrebbe sembrare che, al pari delle vedove aventi precedenza, anche le diaconesse facciano parte del clero. Ma non si parla mai di ordinazione o di designazione per le diaconesse, le quali fanno la comunione con il popolo, in testa alle donne, dopo i laici di sesso maschile, mentre le vedove la fanno dopo i diaconi, prima dei lettori e dei suddiaconi ${ }^{93}$. Come osserva R. Gryson nel Testamento di Nostro Signore Gesu Cristo il rapporto gerarchico tra le vedove e le diaconesse è rovesciato rispetto alle Costituzioni apostoliche $e^{94}$. Secondo le Costituzioni le vedove devono essere soggette alle diaconesse ${ }^{95}$, secondo il Testamento le vedove sono preposte alla sorveglianza delle diaconesse ${ }^{96}$.

Poiché nel Testamento di Nostro Signore Gesù Cristo le vedove rivestono le principali funzioni delle diaconesse delle Costituzioni, il ministero di queste ultime nel Testamento si riduce pressoché a niente. Il fatto che il loro posto in chiesa sia vicino alla porta, potrebbe far pensare che esse

${ }^{89}$ Cfr. R. Gryson, op. cit., p. 127-128.

${ }^{90}$ Cfr. Testamento di Nostro Signore Gesu Cristo I, 19, 7, I. E. Rahmani, Testamentum D. N. Jesu Christi, Magonza 1899, p. 26-27. L'edizione del Rahmani verrà di seguito abbreviata in Rahmani.

${ }_{91}$ Cfr. ibid., I, 23, 1, Rahmani, p. 36-37.

92 Cfr. ibid., I, 35, 5, Rahmani, p. 86-87.

${ }^{93}$ Cfr. ibid., I, 23, 14, Rahmani, p. 46-47.

${ }^{94}$ Cfr. R. Gryson, op. cit., p. 136-137; J. Daniélou, Le ministère des femmes dans l'Église ancienne, "La Maison-Dieu" 61 (1960), p. 83; A. Borras-B. Pottier, La grazia del diaconato. Questioni attuali a proposito del diaconato latino, Assisi 2005, p. 174.

${ }_{95}$ Cfr. Costituzioni apostoliche III, 8, 1, DCA I, 197.

${ }^{96}$ Cfr. Testamento di Nostro Signore Gesù Cristo I, 40, 2, Rahmani, p. 96-97. 
abbiano l'incarico di sorvegliare l'entrata delle donne, come nelle Costituzioni, ma da altri passi risulta che è il diacono a badare all'entrata degli uomini e delle donne, senza differenze ${ }^{97}$, e che spetta ai diaconi, aiutati dai lettori e dai suddiaconi, mantenere l'ordine tra le donne nell'assemblea $^{98}$. Il diacono dice ad alta voce il nome della diaconessa che arriva in ritardo, così come fa per i laici ${ }^{99}$. Infine, la sola funzione che il Testamento di Nostro Signore Gesu Cristo attribuisce in modo chiaro alle diaconesse è quella di portare la comunione alle gestanti che, per il loro stato di salute, non possono recarsi in chiesa il giorno di Pasqua ${ }^{100}$.

\subsection{LA LEGISLAZIONE TEODOSIANA E POST-TEODOSIANA}

Il 21 giugno 390 la legge di Teodosio, revocata il 23 agosto successivo, fissa a sessanta anni l'età di ammissione al ministero delle diaconesse. In questo modo viene applicato alle diaconesse ciò che la $1 \mathrm{Tm} 5$, 9-10 dice riguardo alle vedove ${ }^{101}$. La condizione dell'età sarebbe motivata dalla preoccupazione di evitare che la presenza di donne più giovani in seno a questo gruppo sia causa di defezioni che recherebbero pregiudizio alla reputazione della Chiesa ${ }^{102}$. In seguito il testo proibisce formalmente alla donna divenuta diaconessa di far lasciato di qualsiasi parte dei beni della sua famiglia ad una chiesa, a un chierico, a un povero, e dichiara nulla ogni disposizione testamentaria di questo genere ${ }^{103}$. Questa seconda parte della legge provocò forti proteste e perciò fu revocata due mesi più tardi ${ }^{104}$.

Nel 434 una legge di Teodosio II prescriveva che se un chierico di qualunque grado, un religioso o una religiosa morivano, senza lasciare parenti o moglie, i beni dovevano essere lasciati alla chiesa o al monastero

${ }^{97}$ Cfr. ibid., I, 36, 1-4, Rahmani, p. 88-91.

${ }_{98}$ Cfr. ibid., II, 19, 1, Rahmani, p. 138-139.

${ }_{99}$ Cfr. ibid., I, 36, 4, Rahmani, p. 90-91.

${ }_{100}$ Cfr. ibid., II, 20, 7, Rahmani, p. 142-143.

101 Cfr. Codice Teodosiano 16, 2, 25, Th. Mommsen-P.M. Meyer, Theodosiani libri XVI cum constitutionibus Sirmondianis et leges Novellae ad Theodosianum pertinentes, vol. 1-2, Berlin 1905, p. 842-843. L'edizione del Mommsen-Meyer verrà di seguito abbreviata in Mommsen.

102 Cfr. Sozomeno, Storia eccelsiastica 7, 16, 1-10, GCS 50, 322, 17-324, 9.

103 Cfr. Codice Teodosiano 16, 2, 27, Mommsen, 1-2, p. 843-844.

${ }^{104}$ Cfr. ibid., 16, 2, 28, Mommsen, 1-2, p. 844. 
cui apparteneva il defunto, fatta riserva soltanto per quei beni sui quali il fisco, i patroni o le curie avanzavano dei diritti. Nel numero dei chierici elencati nel testo della legge figura la diaconessa, citata immediatamente dopo il diacono, prima del suddiacono ${ }^{105}$. In questa legge l'imperatore si esprimeva al contrario della legge del 21 giugno 390.

\subsection{LA LEGISLAZIONE DI GIUSTINIANO}

Dalla legislazione di Giustiniano appare chiaramente che le diaconesse sono annoverate tra i chierici. Nella novella 3, che porta la data del 16 marzo 535, indirizzata ad Epifanio arcivescovo di Costantinopoli, l'imperatore propone di porre rimedio alla situazione creata dall'immigrazione in massa di chierici che vengono a gravare in modo insostenibile sul bilancio delle chiese della capitale ${ }^{106}$. Perciò determina il numero di chierici che potranno essere assegnati al servizio di ciascuna di quelle chiese e, in particolare, della cattedrale di Santa Sofia. Quest'ultima non potrà avere più di 60 preti, 100 diaconi, 40 diaconesse, 90 suddiaconi, 110 lettori e 25 cantori, in tutto cioè 425 chierici, oltre a 100 portieri ${ }^{107}$. Quando le leggi di Giustiniano enumerano i diversi gradi del clero, le diaconesse sono associate ai diaconi con la formula "i diaconi di sesso maschile e femminile" e prendono il posto davanti ai suddiaconi, ai lettori e ai cantori, come nella legge di Teodosio del $434^{108}$. Quando i membri della gerarchia del clero non sono enumerati ed il testo parla semplicemente di chierici o di clero in generale, più di una volta la menzione delle diaconesse viene aggiunta espressamente, come se non fosse abbastanza chiaro che esse fossero comprese nei termini generali di "clero" e di "chierici"109.

Nella legislazione di Giustiniano le diaconesse sono assimiliate ai chierici sotto molti punti di vista. Come loro, esse ricevono al momento dell'entrata in funzione, un'ordinazione designata con i termini "cheiro-

105 Cfr. ibid., 5, 3, 1, Mommsen, 1-2, p. 220.

106 Cfr. J. Pargoire, L'Église byzantine de 527 à 847, Paris 1905², p. 60-61.

${ }_{107}$ Cfr. Novellae constitutiones Iustiniani 3, 1, 1, R. Schoell-G. Kroll, Novellae constitutiones Iustiniani, Berlin 19285 , 21, 3-12. L'edizione del Schoell-Kroll verrà di seguito abbreviata in Schoell-Kroll. Le traduzioni del testo saranno proprie.

${ }^{108}$ Cfr. Novellae constitutiones Iustiniani 3, prol., Schoell-Kroll, 19, 16-17.

109 Cfr. Codex Iustiniani 1, 3, 45, 9, P. Krueger, Codex Iustiniani, Berlin 192910, 32. L'edizione del Krueger verrà di seguito abbreviata in Krueger. 
tonia" ("ordinazione"), "cheirotonein" ("ordinare") ${ }^{110}$. Tale ordinazione, come nel caso degli altri chierici, non può essere "indipendente". Ogni diaconessa deve essere aggregata ad una chiesa, secolare o regolare ${ }^{111}$. L'età minima per l'ordinazione al diaconato femminile, che una legge del Codice teodosiano (390) fissava a sessanta anni, che le assimilava alle vedove di $1 \mathrm{Tm}$, è riportata da Giustiniano dapprima a cinquanta anni ${ }^{112}$, ed infine a quaranta ${ }^{113}$. Le candidate devono essere vergini o vedove di un unico marito ${ }^{114}$. Un'opportuna inchiesta deve confermare, che la loro vita, dal punto di vista della castità, sia stata sempre al di sopra di ogni sospetto ${ }^{115}$.

Dopo l'ordinazione, le diaconesse sono tenute alla continenza perfetta, come i chierici maggiori. Quest'obbligo deve essere solennemente ricordato al momento dell'ordinazione, in presenza delle loro consorelle riunite insieme, con lo scopo di ispirare in tutte un salutare timore. Come appariva già nel canone 44 di Basilio e nel canone 15 del Concilio di Calcedonia, il fondamento dell'obbligo consiste non in un impegno personale, ma in una consacrazione risultante dall'ordinazione stessa. La trasgressione viene dunque percepita non come un'infedeltà, ma come una profanazione. Così le sanzioni che colpiscono le ree possono essere molto pesanti. Secondo la novella 6, che non prevede circostanze attenuanti, le diaconesse che hanno disonorato la loro ordinazione sono passibili di pena capitale, come avveniva anche per le vestali infedeli, e i loro beni dati alla chiesa o al monastero a cui erano legate ${ }^{116}$.

Le diaconesse che non risiedono in un monastero devono abitare sole o in compagnia del padre o della madre o dei loro figli, oppure dei loro fratelli o zii con i quali non si può pensare che abbiano relazioni peccaminose. Colei che non osservasse questa regola verrebbe deposta. La novella 6 minaccia la inosservante ed il suo complice con le stesse pene che colpiscono le diaconesse fornicatrici e i loro seduttori ${ }^{117}$. Secondo la

${ }_{110}$ Cfr. Novellae constitutiones Iustiniani 6, 6, Schoell-Kroll, 18, 18; 3, prol., Schoell-Kroll, 18, 26; 131, 13, Schoell-Kroll, 662, 27.

${ }^{111}$ Cfr. Codex Iustiniani 1, 3, 53, 3-4, Krueger, 37; Novellae constitutiones Iustiniani 6, 6, Schoell-Kroll, 44, 4-10.

${ }^{112}$ Cfr. Novellae constitutiones Iustiniani 6, 6, Schoell-Kroll, 43, 27-32.

${ }_{113}$ Cfr. ibid., 123, 13, Schoell-Kroll, 604, 19-21.

${ }^{114}$ Cfr. ibid., 6, 6, Schoell-Kroll, 43, 33-34.

${ }_{115}$ Cfr. ibid., 6, 6, Schoell-Kroll, 43, 35-36.

116 Cfr. ibid., 6, 6, Schoell-Kroll, 44, 30-45, 13.

117 Cfr. ibid., 6, 6, Schoell-Kroll, 44, 10-20 e 25-29. 
novella 123 la colpevole deve essere esclusa dal ministero, privata dei suoi beni e relegata in un convento ${ }^{118}$.

Le diaconesse, come i chierici, godono di una sorta di privilegio del foro, nel senso che colei che avesse una lite con un'altra deve prima portare la causa davanti al vescovo e solo in seconda istanza, se la sentenza episcopale non fosse di proprio gradimento, può proporre appello al giudice civile ${ }^{119}$. Le diaconesse vengono mantenute dalla Chiesa. Esse figurano anche tra coloro che possono essere costituiti beneficiari di una rendita in memoria di un defunto ${ }^{120}$. Inoltre le diaconesse, come i chierici, se muoiono senza legittimi eredi, i loro beni vanno alla chiesa a cui erano legate in forza della loro ordinazione ${ }^{121}$.

Circa le funzioni delle diaconesse, la legislazione di Giustiniano non fornisce informazioni, e non fa meraviglia che sia così, dato il carattere particolare di questa documentazione. La novella 6 indica che le diaconesse esercitano un ministero nella celebrazione del battesimo e, in termini molto vaghi, che "esse sono presenti nelle altre sacre funzioni che abitualmente sono da loro compiute nei venerabili ministeri"122. Questa formula non si capisce bene. Riguardo al compito svolto dalle diaconesse nel battesimo, viene subito da pensare a quegli interventi destinati a salvare la convenienza e il pudore delle donne durante le unzioni e l'immersione battesimale, ciò che si è visto in altri documenti già menzionati.

\subsection{EPIFANIO DI SALAMINA}

Secondo Epifanio di Salamina nella Chiesa vi sono le diaconesse, ma ad esse non competono le azioni sacerdotali. Le diaconesse, come i diaconi, prestano solo un servizio. Il loro ministero, che trova applicazione presso altre donne, ha lo scopo di salvare la decenza, evitando che gli uomini che compiono le funzioni sacre vedano il corpo femminile nel momento in cui deve essere denudato, sia nel battesimo, sia in occasione delle cure prestate alle inferme. Epifanio ricorda pure che, secondo la Sacra Scrittura, non è permesso alla donna di parlare in chiesa, né

\footnotetext{
118 Cfr. ibid., 123, 30, Schoell-Kroll, 616, 17-32.

${ }_{119}$ Cfr. ibid., 123, 21, Schoell-Kroll, 609, 17-611,9.

${ }^{120}$ Cfr. Codex Iustiniani 1, 3, 45, 9, Krueger, 32.

${ }^{121}$ Cfr. Novellae constitutiones Iustiniani 131, 13, Schoell-Kroll, 662, 23-27.

${ }^{122}$ Ibid., 6, 6, Schoell-Kroll, 44, 1-4.
} 
di innalzarsi al di sopra dell'uomo ${ }^{123}$. Le diaconesse, comunque, fanno parte dell'"ordine ecclesiastico", cioè del clero. Nell'enumerazione dei vari ordini, che si trova nel capitolo 21 del Compendio della fede, figura soprattutto la gerarchia sacerdotale, che comprende vescovi, presbiteri, diaconi e suddiaconi ${ }^{124}$. Seguono nell'ordine i lettori, che non fanno parte di questa "gerarchia sacerdotale"125, e le diaconesse ${ }^{126}$. Infine sono menzionati gli esorcisti, gli interpreti e gli ostiari ${ }^{127}$. Gli obblighi imposti alle diaconesse in materia di matrimonio sono gli stessi di quelli dei membri della "gerarchia sacerdotale". Esse devono essere vergini, o vedove di un solo marito, oppure devono aver vissuto il matrimonio nella continenza. Epifanio afferma che le diaconesse sono "costituite" ("kathistasthai") per il servizio concernete le donne ${ }^{128}$. Nella Lettera a Giovanni di Gerusalemme, che si è conservata soltanto nella traduzione latina, scrive però: "Non ho mai ordinato ("ordinavi") delle diaconesse per mandarle in altre province"129. Il primo termine, quello di Epifanio, "costituite" ("kathistasthai"), non esclude che le diaconesse ricevono un'ordinazione, perché è detto in senso molto generale e non espressamente opposto a "cheirotonein" ("ordinare"), come nella Tradizione apostolica e nei testi derivati. Il secondo termine "ordinavi", d'altra parte, non prova decisivamente che Epifanio ordinasse nel vero senso della parola le diaconesse destinate al ministero da svolgere entro i confini della sua provincia, poiché si sa che il documento in questione è solo una traduzione la cui fedeltà è stata contestata. Tuttavia è probabile che la cosa stia così, poiché la pratica dell'ordinazione delle diaconesse tende a generalizzarsi in Oriente, dopo la prima metà del IV secolo ${ }^{130}$.

\subsection{GIOVANNI CRISOSTOMO}

Giovanni Crisostomo ha trattato del ministero delle donne soprattutto nelle sue omelie sulla Sacra Scrittura. Al capitolo 16, 1-2 della

${ }^{123}$ Cfr. Epifanio di Salamina, Compendio della fede 21, 10, GCS 37, 522, 18-20.

${ }^{124}$ Cfr. ibid., 21, 7-8, GCS 37, 522, 18-20.

${ }^{125}$ Cfr. ibid., 21, 9, GCS 522, 13-18.

${ }^{126}$ Cfr. ibid., 21, 10, GCS 522, 18-22.

${ }_{127}$ Cfr. ibid., 21, 11, GCS 522, 22-25.

${ }^{128}$ Cfr. ibid., 21, 10, GCS 522, 18-19.

${ }^{129}$ Lettera di Epifanio di Salamina a Giovanni di Gerusalemme, in: Girolamo, Lettere

51, 2, 2, CSEL 54, 398, 12-13.

${ }^{130}$ Cfr. R. Gryson, op. cit., p. 154-156. 
Lettera ai Romani, trova il nome di molte donne che l'Apostolo prega i suoi corrispondenti di salutare. La prima di esse è Febe "diaconessa" della Chiesa di Cencre. Crisostomo osserva che l'Apostolo aggiunge al nome di Febe la sua qualifica, il titolo cioè di diaconessa, ma non sente il bisogno di dire qualcosa a proposito dell'istituzione stessa delle diaconesse. Egli si limita semplicemente a rilevare l'eccellenza degli elogi tributati a Febe ${ }^{131}$. $E^{\prime}$ da sottolineare che Crisostomo dedica poca attenzione sul diaconato femminile, nonostante che questa istituzione attraversi in quel periodo la sua epoca d'oro e che egli stesso sia in stretto contatto con molte diaconesse. Si sofferma solo sulle donne di cui parla la $1 \mathrm{Tm}$ 3, 11 nella sezione relativa ai diaconi. Egli non dubita che le donne di cui l'Apostolo esige che siano "degne, non maldicenti, sobrie, fedeli in tutto", si identificano con quelle che hanno la "dignità del diaconato"132.

\subsection{TEODORO DI MOPSUESTIA}

Commentando il passo di $1 \mathrm{Tm}$ 3, 11, Teodoro di Mopsuestia dichiara che non si tratta delle moglie dei diaconi, ma delle donne che esercitano la funzione di diacono. Queste donne devono essere dotate di virtù simili a quelle dei diaconi maschili. In particolare, esse devono essere discrete, perché il loro ministero le porterà a ricevere molte confidenze dalle altre donne e se mancassero di discrezione provocherebbero inevitabilmente litigi e divisioni ${ }^{133}$. Teodoro informa anche, che alcuni esigevano per le diaconesse l'età minima di sessanta anni, prescritta da S. Paolo per l'iscrizione nel numero delle vedove ${ }^{134}$. Loro dicevano, infatti, che l'ordine delle diaconesse è più elevato di quello delle vedove.

\subsection{TEODORETO DI CIRO}

Per Teodoreto di Ciro, come per Giovanni Crisostomo e Teodoro di Mopsuestia, non c'è dubbio che le donne di cui parla $1 \mathrm{Tm} 3,11$ sono C-664 A.

${ }^{131}$ Cfr. Giovanni Crisostomo, Omelie sull'Epistola ai Romani 30, 2, PG 60, 663

${ }^{132}$ Cfr, id., Omelie sulla Prima a Timoteo 11, 1, PG 62, 553 CD.

${ }_{133}$ Cfr. Teodoro di Mopsuestia, Commento sulla Prima a Timoteo, Swete 2, 128, $9-129,12$.

${ }^{134}$ Cfr. ibid., Swete 2, p. 139-140. 
le diaconesse. A tale proposito, fa notare la similitudine tra le esigenze proprie di queste e quelle dei diaconi: " "Allo stesso modo le donne», cioè (donne) diaconi, «degne, non maldicenti, sobrie, fedeli in tutto». Ciò che egli prescrive per gli uomini dei pari in termini pressoché uguali, per le donne. Infatti come vuole che i diaconi siano «degni», così anche quelle (devono essere) «degne». E come ordina a quelli di «non avere due parole», analogamente dice a queste di non essere "calunniatrici». Prescrive a quelli di non essere «grandi bevitori di vino» ed a queste, allo stesso modo, impone di essere "sobrie»" 135 .

Nel commento alla Rm 16,1-2, Teodoreto osserva che la comunità cristiana di Cencre, villaggio nei dintorni di Corinto, aveva assunto una certa importanza perché vi si trovava una diaconessa, Febe. Egli vede in questo fatto un indizio del rapido successo ottenuto dalla predicazione evangelica ${ }^{136}$.

\section{LE DIACONESSE GRECHE DAL IV AL VI SECOLO}

Nei documenti storici è rimasto il ricordo di un certo numero di diaconesse orientali, dal IV al VI secolo. Tra queste la più celebre è certamente Olimpia, diaconessa di Costantinopoli. Ella nacque tra il 360 e il 370 da una illustre famiglia, imparentata con re e imperatori. Nel 384 o 385, fu data in sposa a Nebridio, prefetto di Costantinopoli. Assai presto, però, la morte la privò del marito, prima ancora che avesse perduto la sua verginità. Nobiltà di natali, bellezza, patrimonio, ne facevano uno dei più invidiabili partiti. L'imperatore Teodosio fece del tutto perché accettasse di sposare in seconde nozze un certo Elpidio, membro della famiglia imperiale. Olimpia però rifiutò energicamente tale possibilità. Di conseguenza i suoi beni furono messi sotto sequestro. Ma nel 391 ne ricuperò il pieno diritto di disponibilità e, da allora, moltiplicò le sue elargizioni alla Chiesa e ai poveri. Il vescovo Nettario, in considerazione della sua esemplarità, la ordinò diaconessa, nonostante la giovane età. Olimpia costruì un monastero presso la cattedrale di Santa Sofia e vi portò, per cominciare, le sue cameriere in numero di cinquanta, e con esse la sua parente Elisanzia, unitamente alle sorelle di lei Martiria e Palladia. Anche queste ultime tre furono ordinate dia-

\footnotetext{
135 Teodoreto di Ciro, Commento sulla Prima a Timoteo, PG 82, 809 A.

136 Cfr. id., Commento sull'Epistola ai Romani, PG 82, 217 D - 220 A.
} 
conesse. Il numero delle religiose salì ben presto a duecentocinquanta. Quando Giovanni Crisostomo arrivò a Costantinopoli, non tardò a stringere una profonda amicizia con Olimpia. Costei si sentì onorata di provvedere al suo sostentamento fino alla fine della sua vita. Olimpia fu costretta a lasciare Costantinopoli e morì in esilio a Nicomedia il 25 luglio 408.

Di altre diaconesse, al di fuori del gruppo di Giovanni Crisostomo, le notizie sono molto meno dettagliate ${ }^{137}$.

Esiste per esempio una lettera di San Basilio indirizzata "alle diaconesse, figlie del conte Terenzio", scritta nel 372, con la quale egli le incoraggia a rimanere ferme nella fede al Padre, al Figlio e allo Spirito Santo ${ }^{138}$.

Inoltre Teodoreto di Ciro ha scritto le lettere indirizzate a tre diaconesse: Assia, Casiana e Celerina. Le lettere alle prime due sono di condoglianze. Alla terza Teodoreto ricorda in breve la sua dottrina cristologica, chiedendole di impiegare tutte le sue energie in favore della fede e della pace della Chiesa ${ }^{139}$.

\section{LE FONTI CANONICHE LATINE DAL IV AL VI SECOLO}

Nelle fonti canoniche latine, a partire dalla fine del IV secolo, si parla con una certa frequenza di "diaconesse".Tuttavia non si tratta di una vero e proprio ministero, come in Oriente, ma piuttosto di un titolo onorifico attribuito alle "vedove professe".

\subsection{CAN. 2 DEL CONCILIO DI NIMES}

Secondo R. Gryson è probabile che siano stati i priscillianisti a portare per primi dall'Oriente, nell'intento di costituire una condizio-

${ }^{137}$ Cfr. R. Gryson, op. cit., p. 181-184.

${ }^{138}$ Si tratta della Lettera 105 della corrispondenza di Basilio. Testo e traduzione francese di Y. Courtonne nell'edizione delle Lettere appartenente alla "Collection des Universités de France", vol. II, Paris 1968, p. 6-7.

${ }^{139}$ Cfr. Teodorerto di Ciro, Lettere 48, SC 40, 118; 17, SC 98, 62-64, 101, SC 111, $18-20$. 
ne ufficiale ad alcune delle donne che affluivano nella setta, il titolo di "diaconessa", fino ad allora sconosciuto in Occidente ${ }^{140}$.

Infatti, il Concilio di Nimes (394-396), diretto contro il priscillianismo, condanna l'ammissione delle donne al diaconato: " $E$ ' stato anche riferito ("suggestum est") da alcuni che - cosa contraria alla disciplina apostolica e fino ad oggi sconosciuta -, alcune donne siano state ammesse, non si sa dove, al ministero levitico. La disciplina ecclesiastica non ammette ciò, perché è cosa sconveniente. Una ordinazione del genere, fatta a disprezzo della ragione, deve essere annullata, e bisogna in vigilare che per l'avvenire nessuno più dia prova di tanta audacia"141.

Nel suddetto canone non si tratta di un "suggerimento" dato da qualcuno, ma di "voci" che sono arrivate alle orecchie dei Padri conciliari. "Suggerere" non ha il senso di "suggerire", ma il senso tecnico di "fare rapporto, riferire". Si tratta di fatti già accaduti, sui quali il Concilio esprime un giudizio con estrema energia. Quei fatti non si sono verificati proprio con certezza, e le voci che li riferiscono sono abbastanza vaghe: sembra che alcune donne siano state ammesse al ministero del diaconato ("videantur adsumptae"), ma non si sa nemmeno dove ("nescio quo loco"). Inoltre, i Padri del Concilio non precisano la natura del "ministero levitico" al quale quelle donne vengono ammesse. Probabilmente si tratta di un titolo onorifico, accompagnato indubbiamente da una particolare benedizione liturgica, e non un vero e proprio ministero. Questo, ad ogni modo, è quanto suggeriscono i documenti dei secoli successivi.

\subsection{CAN. 25 DEL PRIMO CONCILIO DI ORANGE}

Una cinquantina di anni più tardi, il can. 25 del Primo Concilio di Orange (441), prescrive: "Non bisogna assolutamente ordinare diaconesse; e se ve ne sono ancora, chinino il capo sotto la benedizione che si dà al popolo"142. Il canone proibisce categoricamente l'ordinazione delle diaconesse. I Padri conciliari non hanno ritenuto opportuno motivare la proibizione. La sola informazione che se ne può ricavare è che le diaco-

${ }^{140}$ Cfr. R. Gryson, op. cit., p. 188.

${ }^{141}$ Concilio di Nimes, can. 2, CCL 148, 50, 14-19. La traduzione del testo è propria.

${ }^{142}$ Concilio di Orange I, can. 25, CCL 148, 84, 102-103. La traduzione del testo è propria. 
nesse in questione pretendevano una posizione di privilegio nella Chiesa, dal momento che si prescrive loro di sottomettersi alla benedizione accordata al popolo. Cosa che fa pensare che i chierici ricevevano una benedizione diversa da quella del popolo o che almeno non chinavano la testa durante la benedizione data al popolo. Le diaconesse facevano come il clero. Il Concilio lo proibisce e dichiara loro che non devono comportarsi in maniera diversa dal popolo. Tutto questo, però, non dice nulla circa la natura di queste diaconesse. In realtà sembra, come osserva R. Gryson, che si tratti di vedove che al momento della loro professione avevano ricevuto una benedizione particolarmente solenne, e questa, con la conoscenza così vaga che si aveva delle istituzioni della Chiesa orientale, $\mathrm{fu}$ assimilata all'ordinazione delle diaconesse in uso in Oriente ${ }^{143}$.

\subsection{CANONI DEL CONCILIO DI ÉPAONE E DEL SECONDO CONCILIO DI ORLÉANS}

Il canone 21 del Concilio di Épaone, dove si riunirono nel 517 i vescovi del regno burgundo dichiara: "Sopprimiamo del tutto nell'intera nostra regione la consacrazione delle vedove che spesso sono chiamate "diaconesse"; se esse vogliono convertirsi, potranno ricevere la sola benedizione di penitenza"144.

Questo significa che ancora, in quel periodo, si consacravano delle vedove con un rito solenne che conferiva loro il titolo di "diaconesse". Si tratta certamente di un rito analogo a quello indicato dal termine "ordinare" nel canone 25 del Concilio di Orange. L'intenzione dei vescovi è che tale pratica sparisca del tutto della loro regione.

Infine, di donne che hanno ricevuto un'ordinazione diaconale si parla nel Secondo Concilio di Orléans nel 533. Il canone 17 stabilisce: “Le donne che fino ad oggi, nonostante il divieto dei canoni, hanno ricevuto la benedizione del diaconato, se è provato che sono tornate nuovamente al matrimonio, siano escluse dalla comunione. Ma qualora, ammonite dal vescovo, riconoscano il proprio errore e mettano fine a questa specie di concubinato, recuperino, dopo aver fatto penitenza, la grazia della comu-

${ }^{143}$ Cfr. R. Gryson, op. cit., p. 190.

${ }^{144}$ Concilio di Épaone, can. 21, CCL 148 A, 29, 163-165. La traduzione del testo è propria. 
nione. $E^{\prime}$ stato inoltre deciso che per l'avvenire non si accorderà a nessuna donna la benedizione diaconale, data la fragilità di quel sesso"145.

Anche questa volta si tratta di vedove. Nonostante il divieto dei canoni dei Concili di Orange e di Épaone ancora si parla delle donne cha hanno ricevuto la "benedizione del diaconato". Come è noto i termini "benedictio" e "consecratio" e i relativi verbi figurano spesso, nei testi dell'epoca, come sinonimi di "ordinatio" e di "ordinare". Ancora una volta, dunque, i canoni hanno per oggetto la stessa specie di rito. La cosa tuttavia è vista da un'angolazione diversa da quella dei canoni precedenti. A Orange e ad Épaone, il rito stesso sembrava ai vescovi un'incongruenza. Vogliono che quell'impegno sia diversamente sanzionato. A Orlèans, invece, il rito appare soprattutto come un qualcosa che comporta un impegno di particolare gravità, impegno che è imprudente consacrare, vista la fragilità femminile. Non è previsto nessun rito sostitutivo. Sembra che si voglia semplicemente rinunciare a riconoscere un impegno del genere.

\section{CONCLUSIONE}

Fin dalle origini del cristianesimo, vi sono donne che svolgono un ruolo importante ed occupano una posizione di privilegio in seno alla comunità cristiana. Paolo Apostolo elogia diverse donne che lo hanno assistito nelle sue fatiche apostoliche. Alcune sono favorite da un carisma profetico. Nessuna, tuttavia, esercita funzioni direttive nella comunità. La sola funzione che le donne abbiano esercitato è il diaconato. Ma devono passare i primi due secoli perché il diaconato femminile assuma carattere di consistenza. Infatti pochissime testimonianze relative a donne "ministre", anteriori alla Didascalia Apostolorum sono sommarie, ambigue e non permettono di affermare la regolare esistenza di un diaconato femminile nei primi due secoli. Lo stesso autore della Didascalia rileva l'utilità del ministero della diaconessa in termini che lasciano credere che si tratti di una novità. I compiti assegnati a tale ministero secondo le diverse fonti sono: la cura delle donne inferme, l'assistenza al battesimo delle donne, l'istruzione delle donne neofite, l'accoglienza delle donne nelle assemblee liturgiche, la mediazione tra le donne e gerarchia ecclesiastica maschile.

${ }^{145}$ Concilio di Orléans II, can. 17, CCL 148 A, 101, 64-69. La traduzione del testo è propria. 
Tuttavia la diaconessa non assiste il vescovo e i sacerdoti all'altare e non aiuta a distribuire la comunione.

A partire del IV secolo, le testimonianze sulle diaconesse si fanno numerose in Oriente, tranne l'Egitto, dove mancano le tracce. All'inizio del IV secolo, ed ancora nei canoni di Basilio, appare chiaramente che le diaconesse non sono considerate come facenti parte del clero. Ma dalla fine dello stesso secolo, esse sono annoverate tra i chierici. Come questi, esse vengono ordinate con l'imposizione delle mani con epiclesi dello Spirito Santo, dietro precise condizioni giuridiche. Devono essere vergini o vedove di un solo marito. Epifanio ammette anche le donne che abbiano vissuto in continenza il loro matrimonio. Un'indagine preventiva deve far fede sulla loro moralità. Infine, è stabilito un limite di età che, dopo qualche incertezza, viene fissato a quaranta anni, condizione che nella pratica ha avuto eccezioni anche celebri. Le diaconesse, peraltro, dopo l'ordinazione sono tenute, in materia di matrimonio, all'osservanza di regole analoghe a quelle dei chierici maggiori. Basilio ed Epifanio, che ancora ammettono donne sposate, esigono da loro la continenza perfetta. In seguito, le diaconesse, vergini o vedove che siano, sono rigorosamente escluse dal matrimonio e ogni trasgressione di tale regola, come anche ogni colpa carnale, è colpita da sanzioni durissime. L'istituzione del diaconato femminile, non ha varcato i confini dell'Oriente. I tentativi fatti nel IV e V secolo per introdurla in Occidente, anche se ridotta a semplice dignità $\mathrm{o}$ al titolo onorifico, non ebbero successo.

Streszczenie. Od początku dziejów chrześcijaństwa kobiety odgrywały ważną rolę i zajmowały uprzywilejowaną pozycję we wspólnocie kościelnej. Żadna jednak nie pełniła funkcji kierowniczych we wspólnocie. Jedynymi funkcjami, które wykonywały były związane z przyjętym urzędem diakonatu. W pierwszych dwóch wiekach diakonat kobiet był prawie nieznany. Stąd, autor Didascali Apostolskich mówił wprost o jego nowości. Według różnych źródeł diakonise opiekowały się chorymi kobietami, towarzyszyły im podczas chrztu, pouczały neofitki, troszczyły się o właściwe przyjęcie kobiet we wspólnocie kościelnej podczas celebracji liturgicznych, pośredniczyły między kobietami, a męską hierarchią kościelną, informując o ich potrzebach duchowych i materialnych. Nie asystowały jednak biskupowi, ani też kapłanom przy ołtarzu oraz nie pomagały w rozdzielaniu komunii świętej. Począwszy od IV wieku świadectwa o diakonisach były coraz liczniejsze na Wschodzie, z wyjątkiem Egiptu, gdzie nie było o nich żadnej wzmianki. Podobnie jak mężczyźni były wyświęcane przez biskupa poprzez włożenie rąk i odmówienie epiklezy w obecności całego duchowieństwa. Diakonat kobiet był rozpowszechniony jedynie w kościołach na Wschodzie. Próby wprowadzenia go w IV i V wieku na 
Zachodzie, nawet jedynie w formie godności lub honorowego tytułu nie zakończyły się większym sukcesem.

Słowa kluczowe: kobieta, diakonat kobiet, Kościół starożytny

Abstract. Female diaconate in the ancient Church

Since the beginning of Christianity women played an important role and occupied a special place in the Church community. However, none of them held leadership roles in ecclesial life. The only ministries that they exercised were those connected with diaconate. In the first two centuries, the diaconate of women was unknown; the author of the Didascalia Apostolorum spoke about it as a novelty. According to the different sources, the deaconesses looked after sick women, accompanied them in baptism, taught the neophytesses, attended to the proper reception of women to the Church community during the liturgical celebrations, mediated between women and the Church's hierarchy, and finally provided assistance to their spiritual and material needs. However, they neither assisted the bishops nor the priests at the altar, and did not help them with the distribution of the Holy Communion. Since the $4^{\text {th }}$ century the presence of deaconesses was more and more evident in the East, except Egypt, where no record of them is to be found. Like men, also women were ordained by the bishop through the imposition of hands and the epiclesis in the presence of the clergy. The diaconate of women existed only in the Christian East. The attempts to introduce it to the West in the $4^{\text {th }}$ and $5^{\text {th }}$ century, even in a form of the office or an honorary title, were not successful.

Key words: woman, female diaconate, Ancient Church 